Canadian Oncology

Nursing Journal

Revue canadienne

de soins infirmiers

en oncologie

Volume 30, Issue 3 • Summer 2020

elSSN: 2368-8076 


\section{Optimisation du rôle de l'infirmière spécialisée en oncologie dans l'unité de soins ambulatoires}

\section{par Andrea Knox}

RÉSUMÉ

Si le rôle de l'infirmière spécialisée en oncologie dans le traitement et la prise en charge des symptômes est bien défini, il n'en va pas de même pour le milieu ambulatoire. Les cas se complexifient, l'incidence du cancer augmente et les régimes de traitement ne cessent d'évoluer; cette situation force l'organisme $B C$ Cancer à réévaluer le modèle de ses unités de soins ambulatoires afin de mieux répondre aux besoins des patients. Le présent projet a pour but de déterminer et de schématiser le rôle exact et les tâches fonctionnelles des infirmières travaillant en unité de soins ambulatoires, puis de les comparer aux compétences spécialisées de linfirmière en oncologie. Une liste de référence des rôles fonctionnels des infirmières ainsi qu'une grille des rôles et compétences ont été élaborées à partir d'observations cliniques et de séances de discussion en groupe. Le présent travail aidera à élucider le rôle des infirmières en milieu ambulatoire et permettra à ces dernières d'exprimer clairement la portée de leur pratique et des compétences spécialisées requises pour répondre au mieux aux besoins des patients dans ce milieu. Les résultats du projet pourront servir à orienter les discussions de planification stratégique et opérationnelle qui visent à améliorer les services de santé prodigués aux patients et à offrir du perfectionnement professionnel au personnel de première ligne. Les approches utilisées dans le cadre $d u$ projet sont également intéressantes pour ceux qui voudraient appliquer des méthodes semblables à leur propre milieu de soins du cancer.

\section{INTRODUCTION ET CONTEXTE}

L es infirmières en oncologie de la BC Cancer interviennent Lauprès de patients atteints de cancer à toutes les étapes de la maladie dans différents milieux d'hospitalisation et de soins ambulatoires. BC Cancer a déjà défini clairement le rôle de l'infirmière spécialisée en oncologie dans l'administration des traitements et la prise en charge des effets secondaires (BC Cancer, 2018); toutefois, le rôle de l'infirmière dans l'unité de soins ambulatoires n'est pas aussi bien circonscrit. La complexité des patients en oncologie suivis en externe, l'incidence croissante du cancer (Canadian Cancer Society/Société canadienne du cancer, 2018) et l'émergence continuelle de nouveaux régimes de traitement forcent $\mathrm{BC}$ Cancer à réévaluer le modèle de ses unités de soins ambulatoires.

\section{AUTEURE}

Andrea Knox, inf. aut., B.Sc.inf, M.Sc.inf., CSIO(C)

Responsable principale de la pratique professionnelle, soins infirmiers BC Cancer - Kelowna

1015 Tataryn Road, Kelowna (Colombie-Britannique) V1X 1N6

250-258-4928

aknox@bccancer.bc.ca

DOI:10.5737/23688076303169179
En 2018, un projet en plusieurs étapes a vu le jour pour comprendre les besoins des patients et le rôle des soins infirmiers dans les unités de soins ambulatoires. La première étape, l'initiative de constitution de l'équipe de soins (Care Team Design Initiative) est entrée en phase pilote pour déterminer les besoins prioritaires en matière de soins des patients en oncologie, de même que les perceptions du personnel infirmier actuellement en poste par rapport à l'étendue effective de la pratique infirmière en contexte clinique (BC Children's Hospital et Women's Hospital, BC Cancer, infirmières chercheuses de l'UBC, 2018). Selon les résultats de l'évaluation réalisée auprès des patients, les besoins sont prévisibles et stables dans le milieu des soins ambulatoires (BC Children's Hospital, Women's Hospital, BC Cancer, infirmières chercheuses de l'UBC, 2018). Cependant, dans leurs réponses au questionnaire sur l'étendue de la pratique infirmière (QÉPI), les infirmières ont révélé que leur pratique était, selon elles, plus restreinte dans certains domaines de soins des unités ambulatoires (BC Children's Hospital, BC Women's Hospital, BC Cancer, infirmières chercheuses de l'UBC, 2018).

Grâce à l'initiative de constitution de l'équipe de soins (Care Team Design Initiative), BC Cancer - Kelowna a pu clairement identifier les besoins des patients et les perceptions de la portée de la pratique par le personnel infirmier, mais de plus amples recherches étaient nécessaires pour mieux définir le rôle des infirmières dans le modèle des soins ambulatoires. À l'heure actuelle, la composition des équipes de personnel et l'optimisation de la portée de pratique des infirmières en milieu ambulatoire varient beaucoup entre les différents centres de cancérologie régionaux. Dans certaines cliniques, on ne trouve que des aides-soignants, tandis que d'autres adoptent des modèles mixtes faisant appel à différentes combinaisons d'aides-soignants, d'infirmières auxiliaires autorisées et d'infirmières autorisées. L'absence de normalisation d'un centre à l'autre a entraîné un manque de clarté, tant pour ce qui touche la discipline infirmière que l'équipe interdisciplinaire. La définition et l'optimisation du rôle des soins infirmiers en milieu ambulatoire permettront de bien circonscrire les interventions des infirmières, de même que la portée de leur pratique et les compétences spécialisées requises pour offrir des soins sûrs et de qualité aux patients et aux familles.

\section{BUT}

En Colombie-Britannique, le BC College of Nursing Professionals (BCCNP, 2018a-d) donne une définition plutôt large de la portée de pratique des infirmières autorisées et des infirmières auxiliaires en se fondant sur les exigences énoncées dans la législation encadrant les professions de la santé (Health Professions Act, 2018a-b). Les politiques des employeurs et les compétences individuelles viennent préciser 
cette définition de la portée du rôle des infirmières autorisées et des infirmières auxiliaires. Dans le domaine de l'oncologie, l'Association canadienne des infirmières en oncologie (ACIO/ CANO) a également produit des normes de pratique qui définissent les compétences spécialisées que doivent posséder les infirmières s'occupant de patients cancéreux. S'inscrivant dans la deuxième phase du projet, le présent article a pour but de déterminer et de schématiser le rôle exact et les tâches fonctionnelles des infirmières travaillant en unité de soins ambulatoires, puis de les comparer aux compétences spécialisées de l'infirmière en oncologie (Canadian Association of Nurses in Oncology/Association canadienne des infirmières en oncologie [CANO/ACIO], 2006). Ce projet d'amélioration de la qualité s'inscrit à la suite de l'initiative de constitution de l'équipe de soins (Care Team Design Initiative) et servira de fondement aux prochaines étapes du travail, notamment pour la pondération de la consommation des ressources et la planification de la formation spécialisée propre au rôle des infirmières en unité de soins ambulatoires. L'article présente les résultats du projet, de la revue de littérature initiale et du travail visant à optimiser le rôle de l'infirmière en oncologie en milieu ambulatoire. La démarche suivie pourrait intéresser d'autres organisations souhaitant mener un projet semblable dans leur milieu.

\section{ÉVALUATION DU PROJET PAR L'ARECCI}

À l'heure actuelle, au sein de BC Cancer, il n'existe aucune obligation formelle de procéder à l'examen éthique des projets d'amélioration de la qualité, mais cette absence d'exigences organisationnelles n'exclut en rien le respect des principes éthiques fondamentaux (Hagen et al., 2007). Pour étayer l'examen éthique, le projet a été évalué à l'aide du questionnaire de l'Alberta Research Ethics Community Consensus Initiative (ARECCI). Le score final obtenu avec cet outil était de 2; le projet proposé entrait donc dans la catégorie de risque minimal. Par conséquent, il a été décidé de ne pas soumettre le projet à une approbation éthique, vu son risque minimal et sa forte correspondance avec la définition de l'amélioration de la qualité.

\section{REVUE DE LA LITTÉRATURE}

Une recension des écrits a servi de base pour établir sur des données probantes les fondements du projet d'amélioration de la qualité. L'objectif de la revue de la littérature était d'explorer la recherche et les tendances actuelles entourant la définition du rôle et des compétences de l'infirmière en oncologie en contexte ambulatoire.

\section{Méthodologie}

Les bases de données MedLine, CINAHL et PubMed ont été consultées pour relever les articles récents et pertinents à l'aide des descripteurs MeSH anglais suivants « oncology nursing AND role AND outpatient OR ambulatory AND competency* ». Une brève recherche manuelle des sites Web de l'ACIO/CANO et du British Columbia College of Nursing Professionals (BCCNP) a aussi permis de repérer des documents à l'aide des termes de recherche précédents. Comme l'ACIO/CANO est reconnue à l'échelle nationale et internationale pour son travail dans l'élaboration de normes, de compétences et de ressources propres au domaine de spécialité des soins infirmiers oncologiques, le site Web de l'association a été inclus à la stratégie de recherche. Aussi, le site Web du BCCNP a été dépouillé manuellement pour y relever les écrits pertinents, étant donné que cette organisation fixe les normes et la portée de pratique des infirmières autorisées et des infirmières auxiliaires de la Colombie-Britannique.

Dans ses critères d'inclusion, la recherche documentaire se limitait aux articles complets, en anglais, révisés par les pairs, et publiés entre 2013 et 2018. Il est possible qu'en limitant la recherche, par exemple aux articles complets en anglais seulement, nous ayons exclu incidemment des travaux pertinents, mais le temps et les coûts associés à la traduction des articles rédigés dans une autre langue que l'anglais dépassaient la portée du projet. À noter aussi que, vu l'exclusion des travaux antérieurs à 2013, il est possible que des articles intéressants sur le rôle de l'infirmière en oncologie en soins ambulatoires aient été manqués.

\section{Résultats}

La recherche documentaire préliminaire des sites Web et bases de données a permis de recenser 131 articles, ce nombre ayant été réduit à 115 après retrait des doublons. Après un triage initial par titres et résumés, 96 articles ont été exclus. Les 19 articles restants ont ensuite été lus en entier pour en vérifier la pertinence, et 3 autres articles ont alors été écartés. En fin de compte, 15 articles répondant aux critères d'inclusion ont été retenus; les devis étaient variés : recherche quantitative et modèles à méthode mixte (Azar et al., 2017; BC Children's Hospital, BC Women's Hospital, BC Cancer, infirmières chercheuses de l'UBC, 2018; Berglund et al., 2015; Kaunonen, Salin et Aalto, 2015; Lee, Doran, Tourangeau et Fleshner, 2014), sommaires exécutifs (Haas, Vlasses et Havey, 2016; More, 2017), rapport d'un groupe d'experts (Haas, Swan, et Haynes, 2013), normes professionnelles et normes de pratique (BCCNP, 2018a; BCCNP, 2018b; CANO/ACIO, 2006; CANO/ACIO, 2017; CANO/ACIO, 2018), et documents relatifs à la portée de la pratique des infirmières autorisées et des infirmières auxiliaires de la Colombie-Britannique (BCCNP, 2018c; BCCNP, 2018d).

\section{Constats}

Comme la revue de littérature n'a retenu que cinq recherches formelles comparant les compétences des infirmières en oncologie à leur rôle dans les soins ambulatoires, nous avons inclus à notre projet une combinaison d'études empiriques et d'articles théoriques pour brosser un portrait représentatif des données scientifiques actuelles. Trois grands thèmes se dégagent de la littérature sur le rôle de l'infirmière en oncologie en soins ambulatoires : les dimensions des soins centrés sur le patient, la pratique professionnelle des soins infirmiers oncologiques, et les soins infirmiers dans l'équipe interdisciplinaire.

\section{Les dimensions des soins centrés sur le patient}

Ce premier thème regroupe les normes de soins centrés sur le patient, ainsi que les perceptions des soins par 
les patients (Azar et al., 2017; Berglund et al., 2015; CANO/ ACIO, 2006; Haas, Swan et Haynes, 2013; Haas, Vlasses et Havey, 2016; More, 2017). La prestation de services centrés sur le patient constitue une norme fondamentale de la pratique professionnelle des infirmières autorisées et des infirmières auxiliaires de la Colombie-Britannique, et il est clair que les infirmières doivent travailler avec d'autres membres de l'équipe interdisciplinaire au besoin pour prodiguer des soins qui sont dans le meilleur intérêt des patients (BCCNP, 2018a; BCCNP, 2018b). Pour l'infirmière spécialisée en oncologie, l'ACIO/CANO a énoncé neuf normes supplémentaires qui exposent clairement les soins auxquels les patients et les familles ont droit tout au long de la trajectoire du cancer (tableau 1) (CANO/ACIO, 2006).

\begin{tabular}{|c|c|}
\hline \multicolumn{2}{|c|}{$\begin{array}{l}\text { Tableau 1. Normes de soins de l'ACIO/CANO : Adaptation } \\
\text { du document des Normes de pratique et compétences pour } \\
\text { l'infirmière spécialisée en oncologie (2006) de l'ACIO/CANO }\end{array}$} \\
\hline \multicolumn{2}{|c|}{\begin{tabular}{l|l}
$\begin{array}{l}\text { Soins personnalisés } \\
\text { et holistiques }\end{array}$ & $\begin{array}{l}\text { Les patients atteints de cancer et leur } \\
\text { famille ont droit à des soins personnalisés et } \\
\text { holistiques. }\end{array}$
\end{tabular}} \\
\hline \\
\hline $\begin{array}{l}\text { Autodétermination } \\
\text { et prise de décisions }\end{array}$ & $\begin{array}{l}\text { Les patients atteints de cancer et leur } \\
\text { famille ont le droit d'accéder à l'information } \\
\text { concernant leurs soins et de prendre ensuite } \\
\text { les décisions qui s'imposent. }\end{array}$ \\
\hline $\begin{array}{l}\text { Savoir naviguer } \\
\text { dans le système }\end{array}$ & $\begin{array}{l}\text { Les patients atteints de cancer et leur } \\
\text { famille ont droit à une aide pour cheminer } \\
\text { dans le système de santé à toutes les étapes } \\
\text { de la lutte contre la maladie. }\end{array}$ \\
\hline $\begin{array}{l}\text { Soins coordonnés } \\
\text { continus }\end{array}$ & $\begin{array}{l}\text { Les patients atteints de cancer et leur } \\
\text { famille ont droit à des soins coordonnés de } \\
\text { la part des différents prestataires, et ce, à } \\
\text { toutes les étapes de la lutte contre le cancer. }\end{array}$ \\
\hline $\begin{array}{l}\text { Relation } \\
\text { thérapeutique basée } \\
\text { sur le soutien }\end{array}$ & $\begin{array}{l}\text { Les patients atteints de cancer et leur famille } \\
\text { ont droit, tout au long de l'expérience du } \\
\text { cancer, à une relation thérapeutique basée } \\
\text { sur le soutien avec des professionnels de la } \\
\text { santé ayant les connaissances nécessaires. }\end{array}$ \\
\hline $\begin{array}{l}\text { Soins fondés sur des } \\
\text { données probantes }\end{array}$ & $\begin{array}{l}\text { Les patients atteints de cancer et leur } \\
\text { famille ont droit à des soins fondés sur la } \\
\text { recherche et les pratiques exemplaires. }\end{array}$ \\
\hline Soins professionnels & $\begin{array}{l}\text { Les personnes atteintes de cancer et leur } \\
\text { famille ont droit à des soins professionnels et } \\
\text { éthiques respectant les obligations légales. }\end{array}$ \\
\hline Leadership & $\begin{array}{l}\text { Les personnes atteintes de cancer et leur } \\
\text { famille ont droit à des soins prodigués au sein } \\
\text { d'un système de santé dont le leadership } \\
\text { professionnel est axé sur les patients. }\end{array}$ \\
\hline
\end{tabular}

Pour faciliter la coordination des soins et la gestion de la transition dans le milieu ambulatoire, Haas, Swan et Hayes (2013) ont aussi défini les dimensions des soins aux patients. Du point de vue des patients, la coordination de la continuité des soins est considérée comme particulièrement importante (Berglund et al., 2015). La continuité des soins peut comprendre les besoins en matière d'information, de relations et de gestion des soins - de l'avis des patients, cette continuité est optimale lorsque l'unité ambulatoire compte dans ses rangs une infirmière coordonnatrice ou une infirmière pivot. Azar et collaborateurs (2017) ont mesuré le rapport entre le travail d'équipe et la perception des soins par les patients; la coordination relationnelle, un rôle joué par les infirmières coordonnatrices et axé sur la continuité des soins, a obtenu un score élevé, corrélé à une perception positive des soins. On trouve donc dans la littérature actuelle des données convaincantes pour étayer les dimensions des soins du cancer centrés sur les patients (BCCNP, 2018a; BCCNP, 2018b; CANO/ACIO, 2006; Haas, Swan et Haynes, 2013) qui concordent avec la perception des patients des soins prodigués par les infirmières en oncologie en contexte ambulatoire (Azar et al., 2017; Berglund et al., 2015).

La pratique professionnelle des soins infirmiers oncologiques

Le deuxième thème retenu touchait principalement la pratique professionnelle des infirmières en oncologie. Il comprend plus précisément les compétences requises et les normes de pratique dérivées des normes professionnelles de soins et des dimensions des soins aux patients (BCCNP, 2018a; BCCNP, 2018b; CANO/ACIO, 2006; Haas, Swan et Haynes, 2013). À partir des neuf normes de soins de l'ACIO/CANO (tableau 1), sept normes de pratiques et compétences connexes ont été établies pour l'infirmière spécialisée en oncologie : évaluation globale de la santé, relation thérapeutique basée sur le soutien, gestion des symptômes du cancer et des effets secondaires des traitements, enseignement et encadrement, facilitation de la continuité des soins et savoir naviguer dans le système, prise de décisions et défense des droits du patient, pratique professionnelle et leadership (CANO/ACIO, 2006). Les neuf normes de soins (tableau 1) et les sept normes de pratique ont aussi été intégrées aux normes de l’ACIO/CANO pour les sous-spécialités que constituent la radiothérapie et les traitements systémiques comme la chimiothérapie (CANO/ACIO, 2017; CANO/ ACIO, 2018). Ces documents décrivent les normes de pratiques, la formation et les compétences de perfectionnement professionnel des infirmières qui s'occupent de patients recevant de la radiothérapie ou un traitement systémique au Canada (CANO/ ACIO, 2017; CANO/ACIO, 2018). Haas, Swan et Hayes (2013) ont réuni un groupe d'experts pour déterminer les compétences propres au rôle infirmier en contexte ambulatoire. Bien qu'elles ne soient pas spécifiques à l'oncologie, les neuf dimensions des soins aux patients ainsi retenues correspondaient à des compétences directement liées aux normes de pratique de l'ACIO/ CANO. Cela montre la concordance entre la pratique professionnelle des infirmières en oncologie et la recherche portant sur la pratique professionnelle des infirmières en soins ambulatoires (CANO/ACIO, 2006; CANO/ACIO, 2017; CANO/ACIO, 2018; Haas, Swan et Haynes, 2013). 


\section{Les soins infirmiers et l'équipe interdisciplinaire}

Le troisième thème portait sur la place de l'infirmière en oncologie dans l'équipe interdisciplinaire, plus précisément sur l'importance d'adopter un modèle de soins hautement collaboratifs et offerts par la bonne combinaison de professionnels afin répondre au mieux aux besoins des patients (Azar et al., 2017; ACIO/CANO, 2006; Haas, Swan et Haynes, 2013; Haas, Vlasses et Havey, 2016; Kaunonen, Salin et Aalto, 2015; Lee, Doran, Tourangeau et Fleshner, 2014; More, 2017). En général, les infirmières en oncologie et les médecins sont d'avis que leurs interactions professionnelles en milieu ambulatoire sont de très bonne qualité (Lee, Doran, Tourangeau et Fleshner, 2014) et considèrent le rôle joué par l'infirmière pour faciliter la communication et la coordination des soins au sein de l'équipe interdisciplinaire comme un élément clé d'une bonne communication (Azar et al., 2017; Haas, Swan et Haynes, 2013; Lee, Doran, Tourangeau et Fleshner, 2014; More, 2017). Toutefois, la clarté du rôle de chaque membre de l'équipe constitue un autre facteur important, puisqu'elle permet d'améliorer l'efficacité générale de l'équipe interdisciplinaire (Haas, Swan et Haynes, 2013; Haas, Vlasses et Havey, 2016). Pour atténuer les difficultés que pourrait causer le manque de clarté des rôles, nous recommandons que le personnel infirmier de première ligne participe à l'élaboration du modèle de soins ambulatoires afin d'aider les décideurs à choisir les professionnels de la santé les mieux placés pour répondre aux besoins des patients (Haas, Vlasses et Havey, 2016; Kaunonen, Salin et Aalto, 2015).

\section{Analyse des lacunes}

Le nombre de recherches formelles portant sur l'optimisation du rôle de l'infirmière en oncologie dans les soins ambulatoires est nettement insuffisant. La plupart des articles recensés n'étaient pas des études à proprement parler. Pour guider le travail d'optimisation du rôle proposé pour les infirmières, nous avons consulté les compétences et les normes de pratiques propres au domaine de spécialité des soins infirmiers oncologiques en contexte canadien; elles abondent dans le même sens que les recommandations de la recherche actuelle. En outre, la littérature portant sur le rôle de l'infirmière dans l'équipe interdisciplinaire, sur les dimensions des soins aux patients et sur les perceptions qu'ont les patients des soins infirmiers oncologiques présentant également des éléments similaires. Il était donc pertinent d'approfondir les recherches touchant plus précisément l'optimisation du rôle de l'infirmière en oncologie en milieu ambulatoire. Notre projet d'amélioration de la qualité a été conçu pour combler les lacunes constatées dans la littérature et faire avancer la pratique des infirmières en oncologie de BC Cancer.

\section{CONTEXTE DU PROJET}

Le projet d'optimisation du rôle de l'infirmière en oncologie dans les soins ambulatoires a été réalisé dans un contexte clinique particulier, celui de l'unité de soins ambulatoires, constituée d'une équipe interdisciplinaire formée de personnel administratif, d'infirmières, de pharmaciens, d'omnipraticiens en oncologie, d'infirmières praticiennes (IP), ainsi que de radio-oncologues et d'oncologues médicaux. La clinique compte cinq infirmières auxiliaires et deux infirmières autorisées sur le plancher, dont les fonctions principales sont de contribuer à la gestion des patients et de prodiguer un large éventail de services infirmiers. Les patients qui viennent à la clinique sont soit des nouveaux patients, soit en traitement actif ou en suivi post-traitement. Cent quarante-quatre nouveaux patients et 563 patients en traitement actif ou en suivi sont vus chaque semaine, et les consultations durent entre 20 et 90 minutes. Dans le cadre du projet, les groupes d'intérêt ciblés étaient les patients en oncologie, le personnel infirmier de BC Cancer - Kelowna, l'équipe de direction de BC Cancer - Kelowna, et l'équipe du programme provincial de pratique professionnelle des infirmières (Professional Practice Nursing) de BC Cancer. Le projet a reçu un appui et un financement régional et provincial.

Bien que les modèles de soins et les ressources diffèrent, tous les centres de cancérologie régionaux gèrent une unité de soins ambulatoires; ainsi, les résultats du présent projet pourraient influencer les décisions touchant le fonctionnement de toutes les unités de soins ambulatoires de BC Cancer. Pour éviter un dédoublement du travail, des liens ont été établis avec les intervenants clés des autres établissements de BC Cancer pour faciliter la collaboration aux étapes de planification, de mise en œuvre, d'évaluation et de diffusion du projet. D'autres centres avaient déjà mis en place diverses initiatives en lien avec le modèle de soins de l'unité ambulatoire; toutefois les buts et objectifs du présent projet ont été jugés complémentaires et utiles aux efforts en cours.

\section{FACTEURS FAVORABLES}

En collaboration avec le mentor qui parraine le projet, les facteurs favorables à l'atteinte des objectifs ont été expressément identifiés, puis mis en relation avec le plan général du projet. Le parrainage et soutien du projet par le centre régional était justement l'un de ces facteurs favorables et tangibles ayant facilité l'accès à la clinique de soins ambulatoires et à son personnel. De la même façon, en encourageant les discussions continues sur la portée de pratique et les modèles de soins, le présent projet a également reçu un soutien à l'échelon provincial. Le soutien provincial était essentiel afin de réduire autant que possible toute répercussion négative éventuelle qu'auraient pu entraîner d'autres priorités organisationnelles concurrentes. Les infirmières et les autres membres de l'équipe interdisciplinaire qui travaillent à l'unité de soins ambulatoires ont aussi manifesté un fort désir de clarifier le rôle des infirmières, vu comme un facteur clé pour faciliter la participation du personnel aux activités du projet, comme les groupes de discussion qui avaient pour tâche de valider les propositions. D'autres facteurs positifs préexistants ont été relevés, notamment les normes et les compétences de BCCNP et de l'ACIO/CANO; ces ressources, auxquelles s'ajoutent les résultats du projet pilote de l'initiative de constitution de l'équipe de soins (Care Team Design), ont permis d'orienter et de compléter les travaux.

\section{CONTRAINTES}

Plusieurs obstacles potentiels ont été décelés et des stratégies d'atténuation ont dû être mises en place pour mener le projet 
à terme. Dans la clinique, les difficultés relatives à la charge de travail et à la dotation en personnel étaient considérées comme des barrières pouvant entraver la disponibilité du personnel de première ligne. Dès le début du projet, il s'est avéré essentiel de discuter avec le gestionnaire clinique et le responsable des infirmières de première ligne afin de remplacer le personnel participant aux groupes de discussion. Les priorités organisationnelles concurrentes, comme le projet d'agrément ou de transformation des systèmes cliniques, constituaient également des contraintes évidentes à la réalisation du projet, étant donné le chevauchement des délais de réalisation prévus. Pour éviter de possibles retards, le plan de travail du projet prévoyait un suivi régulier du calendrier et des activités proposées pour toutes les initiatives concurrentes. Ainsi, il était possible de faire preuve de souplesse et d'ajuster les activités et le calendrier du projet au besoin pour éviter les retards.

Autre contrainte : la portée limitée du travail des infirmières auxiliaires en oncologie pourrait avoir influencé négativement la participation de ces dernières au travail de validation des groupes de discussion. La principale stratégie employée pour atténuer cet obstacle potentiel a été de reconnaître la portée limitée de la pratique des infirmières auxiliaires et d'expliquer les résultats attendus pour clarifier la portée du travail et les besoins en formation à moyen terme.

Enfin, la clinique est petite et très achalandée, ce qui peut avoir compliqué les exercices d'observation et de schématisation des processus. Pour réduire le plus possible l'incidence des activités du projet sur l'espace physique, l'horaire provisoire des séances d'observation à la clinique était passé en revue à l'avance pour vérifier que la charge de travail pour le jour prévu était normale et qu'il n'y avait pas de pénurie de personnel. La stratégie d'atténuation consistait donc à réaliser les observations lorsque la charge de travail était « habituelle » et qu'il ne manquait pas de personnel.

\section{MÉTHODOLOGIE}

Les principaux intervenants appelés à participer au projet (c'est-à-dire aux exercices d'observation et aux discussions de groupe) appartenaient au personnel infirmier de première ligne. Les séances d'observation, principale stratégie utilisée pour recueillir l'information nécessaire à l'élaboration de la liste de référence des éléments du rôle fonctionnel, se sont déroulées sur quatre journées. Pendant l'exercice d'observation, nous avons consigné l'organisation du travail du personnel infirmier, ainsi que les activités directement associées aux soins aux patients. Pour schématiser l'organisation du travail, nous avons créé une représentation visuelle des fonctions accomplies par les infirmières pour faciliter l'accueil des nouveaux patients, les traitements actifs et les rendez-vous de suivi (figure 1).

Deux groupes de discussion avec animateur ont été organisés pour commenter et valider l'information recueillie. Le premier groupe de discussion a évalué, peaufiné et validé la liste de référence préliminaire des éléments du rôle fonctionnel préparée à partir de l'exercice d'observation et de schématisation des processus. Après les discussions du premier groupe, la liste de référence validée des rôles fonctionnels a été comparée aux normes de BCCNP et de l'ACIO/CANO pour vérifier que les activités proposées correspondaient bien aux pratiques exemplaires recommandées pour le soin des patients en contexte oncologique (BCCNP, 2018a; BCCNPb, 2018; BCCNP, 2018c; BCCNP, 2018d; CANO/ACIO, 2006; CANO/ACIO, 2017; CANO/ACIO, 2018). Un second groupe, formé de personnel de première ligne, a ensuite évalué et validé la grille proposée des rôles et compétences.

Des guides de questions ont été élaborés pour encadrer le contenu à couvrir (tableau 2) et ainsi faciliter l'animation des groupes de discussion. Un court sondage électronique,

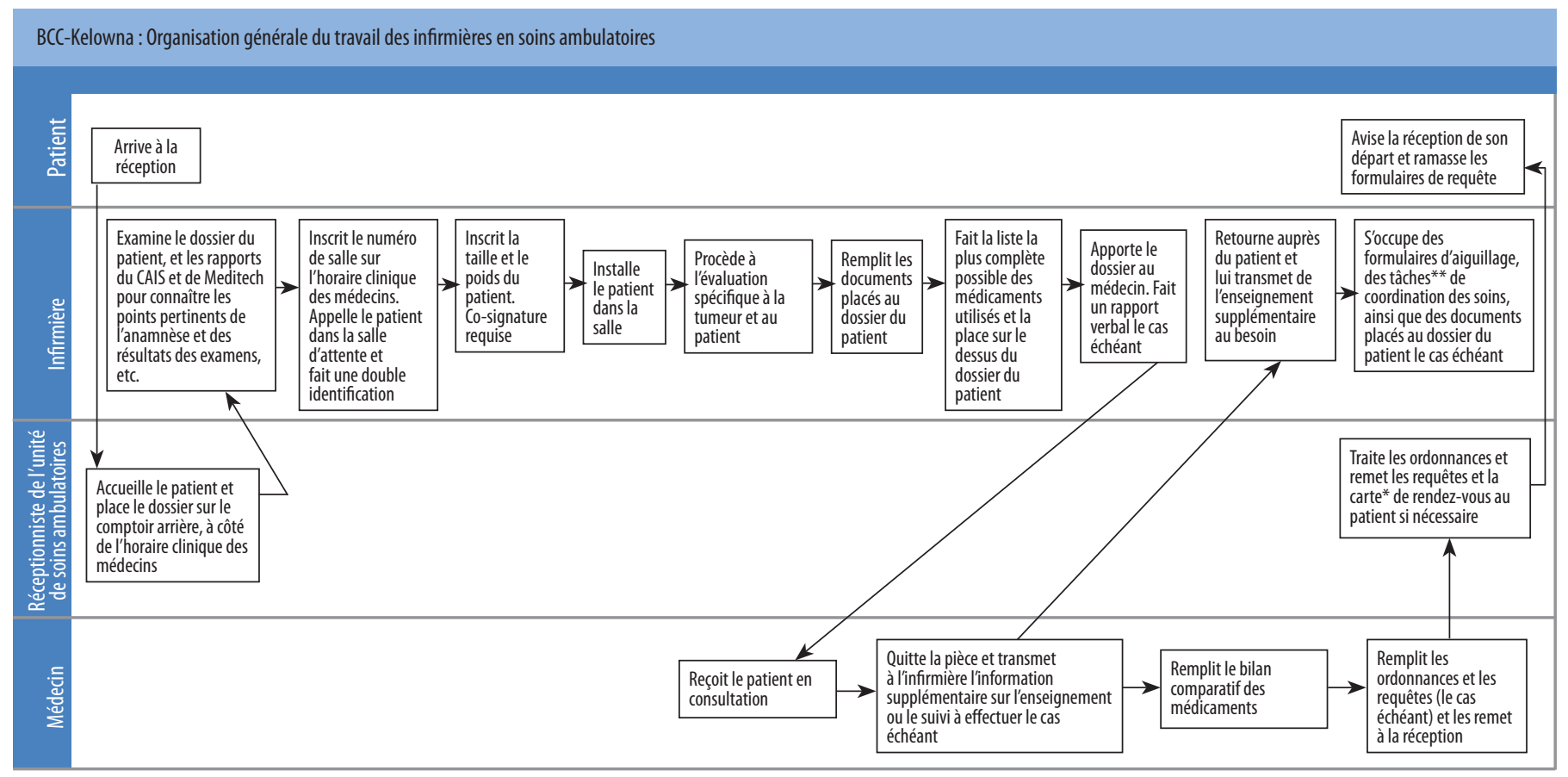

Figure 1. Diagramme de l'organisation du travail des infirmières en unité ambulatoire 


\section{Tableau 2. Guides d'entrevue}

\section{Guide d'entrevue pour le groupe de discussion 1}

Objectif : Réviser et valider la liste de référence des composantes du rôle fonctionnel avec le personnel infirmier de l'unité de soins ambulatoires.

\section{Introduction}

- Faire les présentations des membres du groupe et expliquer l'objectif du groupe de discussion.

- Survoler avec les participants le plan de travail du groupe de discussion.

- Faire un survol de la proposition de liste de référence des éléments du rôle fonctionnel.

1. En unité de soins ambulatoires, dans votre rôle d'infirmière en TS auprès des nouveaux patients, les activités que vous accomplissez actuellement figurent-elles toutes sur la liste proposée?

a. Si non, quelles sont les activités manquantes?

2. Y $\mathrm{a}$-t-il des activités que vous n'accomplissez pas actuellement dans le cadre de votre rôle d'infirmière en unité de soins ambulatoires pour les nouveaux patients à cause d'un manque de temps ou de ressources, et qui devraient être inscrites sur cette liste, selon vous?

a. Si oui, quelles activités aimeriez-vous ajouter?

3. En unité de soins ambulatoires, dans votre rôle d'infirmière en RT auprès des nouveaux patients, les activités que vous accomplissez actuellement figurent-elles toutes sur la liste proposée?

a. Si non, quelles sont les activités manquantes?

4. Ya-t-il des activités que vous n'accomplissez pas actuellement dans le cadre de votre rôle d'infirmière en unité de soins ambulatoires pour les nouveaux patients à cause d'un manque de temps ou de ressources, et qui devraient être inscrites sur cette liste, selon vous?

a. Si oui, quelles activités aimeriez-vous ajouter?

5. En unité de soins ambulatoires, dans votre rôle d'infirmière en TS auprès des patients en traitement actif, les activités que vous accomplissez actuellement figurent-elles toutes sur la liste proposée?

a. Si non, quelles sont les activités manquantes?

6. Ya-t-il des activités que vous n'accomplissez pas actuellement dans le cadre de votre rôle d'infirmière en unité de soins ambulatoires pour les patients en traitement actif à cause d'un manque de temps ou de ressources, et qui devraient être inscrites sur cette liste, selon vous?

a. Si oui, quelles activités aimeriez-vous ajouter?

7. En unité de soins ambulatoires, dans votre rôle d'infirmière en TS auprès des patients en suivi, les activités que vous accomplissez actuellement figurent-elles toutes sur la liste proposée?

a. Si non, quelles sont les activités manquantes?

8. Ya-t-il des activités que vous n'accomplissez pas actuellement dans le cadre de votre rôle d'infirmière en unité de soins ambulatoires pour les patients en suivi à cause d'un manque de temps ou de ressources, et qui devraient être selon vous inscrites sur cette liste?

a. Si oui, quelles activités aimeriez-vous ajouter?

9. En unité de soins ambulatoires, dans votre rôle d'infirmière en RT auprès des patients nouvellement en suivi, les activités que vous accomplissez actuellement figurent-elles toutes sur la liste proposée?

a. Si non, quelles sont les activités manquantes?

10. Ya-t-il des activités que vous n'accomplissez pas actuellement dans le cadre de votre rôle d'infirmière en unité de soins ambulatoires pour les patients nouvellement en suivi à cause d'un manque de temps ou de ressources, et qui devraient être selon vous inscrites sur cette liste?

a. Si oui, quelles activités aimeriez-vous ajouter?

11. Ya-t-il des activités que vous accomplissez comme infirmière en unité ambulatoire qui ne sont pas directement liées à un type particulier de patients et qui ne figurent pas sur la liste proposée?

a. Si oui, quelles activités aimeriez-vous ajouter?

12. Ya-t-il des activités qui ne sont pas liées à un type particulier de patients et que vous n'accomplissez pas actuellement dans le cadre de votre rôle d'infirmière en unité de soins ambulatoires à cause d'un manque de temps ou de ressources, et qui devraient être selon vous inscrites sur cette liste?

a. Si oui, quelles activités aimeriez-vous ajouter?

13. Ya-t-il des activités de la liste proposée qui, selon vous, ne font pas partie du rôle de l'infirmière en unité de soins ambulatoires et qui devraient être retirées?

a. Si oui, lesquelles?

14. Selon vous, la liste rend-elle compte avec exactitude du rôle de l'infirmière en unité de soins ambulatoires pour les traitements systémiques?

15. Selon vous, la liste rend-elle compte avec exactitude du rôle de l'infirmière en unité de soins ambulatoires pour les traitements de radiothérapie?

16. Avez-vous d'autres commentaires, questions ou suggestions à formuler? 


\section{Guide d'entrevue pour le groupe de discussion 2}

Objectif : Valider la grille des rôles et compétences complétée auprès du personnel infirmier de l'unité de soins ambulatoires

\section{Introduction}

- Faire les présentations des membres du groupe et expliquer l'objectif du groupe de discussion.

- Survoler avec les participants le plan de travail du groupe de discussion.

- Réviser la liste de référence des éléments du rôle fonctionnel préalablement validée.

- Passer en revue les normes et les compétences définies par l'ACIO/CANO pour l'infirmière spécialisée en oncologie.

- Donner un aperçu de la grille proposée des rôles et des compétences.

1. En passant en revue les éléments du rôle fonctionnel de l'infirmière pour les nouveaux patients en traitement systémique, les compétences de l'ACIO/CANO définies pour chaque activité correspondante de la liste des éléments du rôle fonctionnel sontelles appropriées?

a. Si non, quelle modification de la grille recommanderiez-vous pour qu'elle rende compte plus fidèlement des compétences de l'ACIO/CANO?

2. En passant en revue les éléments du rôle fonctionnel de l'infirmière auprès des patients en traitement systémique actif, diriez-vous que les compétences de l'ACIO/CANO définies pour chaque activité correspondante de la liste des éléments du rôle fonctionnel sont appropriées?

a. Si non, quelle modification de la grille recommanderiez-vous pour qu'elle rende compte plus fidèlement aux compétences de I'ACIO/CANO?

3. 3. En passant en revue les éléments du rôle fonctionnel de l'infirmière auprès des patients en suivi après un traitement systémique, diriez-vous que les compétences de l'ACIO/CANO définies pour chaque activité correspondante de la liste des éléments du rôle fonctionnel sont appropriées?

a. Si non, quelle modification de la grille recommanderiez-vous pour qu'elle rende compte plus fidèlement aux compétences de l'ACIO/CANO?

4. En passant en revue les éléments du rôle fonctionnel de l'infirmière auprès des nouveaux patients en radiothérapie, diriez-vous que les compétences de l'ACIO/CANO définies pour chaque activité correspondante de la liste des éléments du rôle fonctionnel sont appropriées?

a. Si non, quelle modification de la grille recommanderiez-vous pour qu'elle rende compte plus fidèlement aux compétences de l'ACIO/CANO?

5. En passant en revue les éléments du rôle fonctionnel de l'infirmière auprès des patients en suivi après une radiothérapie, diriez-vous que les compétences de l'ACIO/CANO définies pour chaque activité correspondante de la liste des éléments du rôle fonctionnel sont appropriées?

a. Si non, quelle modification de la grille recommanderiez-vous pour qu'elle rende compte plus fidèlement aux compétences de l'ACIO/CANO?

6. Êtes-vous d'accord avec les rôles et les compétences des infirmières retenus dans la grille finale proposée pour faciliter les rendezvous des nouveaux patients en traitement systémique?

7. Êtes-vous d'accord avec les rôles et les compétences des infirmières retenus dans la grille finale proposée pour faciliter les rendezvous des nouveaux patients en radiothérapie?

8. Êtes-vous d'accord avec les rôles et les compétences des infirmières retenus dans la grille finale proposée pour faciliter les rendezvous des patients en traitement systémique actif?

9. Êtes-vous d'accord avec les rôles et les compétences des infirmières retenus dans la grille finale proposée pour faciliter les rendezvous des patients en traitement actif de radiothérapie?

10. Êtes-vous d'accord avec les rôles et les compétences des infirmières retenus dans la grille finale proposée pour faciliter les rendezvous des patients en suivi après un traitement systémique?

11. Êtes-vous d'accord avec les rôles et les compétences des infirmières qui ont été retenus dans la grille finale proposée et qui ne sont pas rattachés à un type particulier de patients?

12. Selon vous, la liste rend compte avec exactitude des normes et compétences de l'ACIO/CANO du rôle de l'infirmière en unité de soins ambulatoires pour les traitements systémiques?

13. Selon vous, la liste rend compte avec exactitude des normes et compétences de l'ACIO/CANO du rôle de l'infirmière en unité de soins ambulatoires pour les traitements de radiothérapie?

14. Avez-vous d'autres commentaires, questions ou suggestions à formuler? 
administré auprès des participants dans la semaine suivant chaque séance, permettait de mesurer la satisfaction générale et la perception de l'efficacité du groupe de discussion. La collecte de données à l'aide de sondages électroniques respectait les politiques et procédures organisationnelles afin de satisfaire à la loi sur l'accès à l'information et la protection de la vie privée de la Colombie-Britannique (BC Government, 2019). Toutefois, l'outil de sondage ayant connu des problèmes techniques, une autre approche a finalement été retenue pour ne pas retarder le projet. Les commentaires des participants ont été recueillis directement, à l'oral.

\section{RÉSULTATS}

Grâce à une planification rigoureuse, les exercices d'observation et de discussion ont pu avoir lieu sans anicroche et le personnel infirmier de première ligne a pu élaborer et valider la liste des éléments du rôle fonctionnel, ainsi que la grille des rôles et des compétences (tableau 3). L'organisation générale du travail a également été schématisée pour comprendre l'influence et les défis d'intégration d'un large éventail de tâches réalisées par les infirmières en contexte clinique (figure 1).

Chaque séance de discussion réunissait de 4 à 6 infirmières de première ligne travaillant dans les cliniques de radiothérapie et de traitement systémique de l'unité de soins ambulatoires. Les infirmières en radiothérapie (RT) ont examiné et validé la liste de référence des éléments du rôle fonctionnel, ainsi que la grille finale des rôles et compétences décrivant les soins de RT de l'unité de soins ambulatoires, pour un total de 69 tâches. De la même façon, les infirmières en traitement systémique (TS) ayant participé aux groupes de discussion ont examiné et validé les mêmes documents pour les TS en unité de soins ambulatoires, pour un total de 58 tâches. Les documents ont ensuite été regroupés en fonction des tâches de préparation clinique et des types de rendez-vous (nouveau patient, traitement actif et suivi) de manière à englober les différents besoins des patients sur toute la trajectoire des soins. Le tableau 3 présente un extrait des grilles validées de rôles et compétence pour la radiothérapie et les traitements systémiques.

Selon les commentaires recueillis à l'oral directement auprès du personnel participant, les méthodes employées ont été considérées comme efficaces et appropriées pour faciliter leur participation à l'exercice fondamental que constituait le présent projet. Dans l'ensemble, le travail réalisé a permis de comprendre l'état actuel du rôle et de l'organisation $\mathrm{du}$ travail des infirmières, de peaufiner et de valider la liste de référence des éléments du rôle fonctionnel, et de créer une grille exhaustive des rôles et compétences à partir des normes de pratiques provinciales encadrant les soins infirmiers spécialisés.

\section{Limites}

Le projet comportait certaines limites, notamment dans sa portée générale, le contexte de l'unité de soins ambulatoires, et la capacité à généraliser le contenu des documents produits. Au départ, le projet visait l'optimisation du rôle de l'infirmière en contexte ambulatoire. Ce point est très pertinent et le sujet est très peu abordé dans la littérature; toutefois, les entretiens directs avec le personnel de première ligne a permis de constater la volonté d'optimiser aussi le rôle de tous les membres de l'équipe de soins, et pas uniquement celui des infirmières. Par conséquent, il est recommandé d'appliquer l'approche employée dans le cadre du projet pour mieux clarifier les rôles des autres professionnels de la santé de l'unité de soins ambulatoires et ainsi optimiser le modèle de l'équipe interdisciplinaire.

Le fait que le projet se déroule principalement à la clinique de $\mathrm{BC}$ Cancer - Kelowna limitait également sa portée générale. Rappelons que les six centres de cancérologie régionaux de $\mathrm{BC}$ Cancer disposent tous d'une unité de soins ambulatoires, mais que leurs modèles de soins et leurs ressources infirmières diffèrent. Les résultats du présent projet pourraient influencer les décisions touchant toutes les unités ambulatoires de BC Cancer, mais les différents modèles en place à l'heure actuelle empêchent de généraliser à tous les établissements la liste de référence des éléments du rôle fonctionnel et la grille des rôles et compétences. À cause de ces différences, les documents du projet devront être validés avant d'être adoptés dans les cinq autres centres pour en assurer la pertinence et l'applicabilité. Le contexte même de l'unité de soins ambulatoires était considéré comme une limite du projet, étant donné que les éléments figurant dans la liste de référence des rôles fonctionnels et dans la grille des rôles et compétences sont communs à d'autres rôles qu'exercent les infirmières en dehors de l'unité de soins ambulatoires de BC Cancer - Kelowna. Comme le désir d'optimiser les rôles de toute l'équipe interdisciplinaire de l'unité de soins ambulatoires qui vient d'être relevé, le personnel infirmier de première ligne s'est aussi montré intéressé à étendre le travail d'optimisation du rôle infirmier à d'autres domaines cliniques. Bien que cette tâche dépasse la portée et les visées du présent projet, elle fait partie des sujets à explorer aux prochaines étapes de la recherche.

\section{Recommandations}

Étant donné la satisfaction rapportée par les infirmières de première ligne et la concordance de l'outil avec les normes professionnelles provinciales et spécialisées, nous recommandons que la grille des rôles et compétences serve de base pour orienter le travail à venir sur la pondération de la consommation des ressources et le développement de la formation destinée aux infirmières. Nous recommandons également que la liste de référence des éléments du rôle fonctionnel et la grille des rôles et compétences soient validées par le personnel infirmier de l'unité de soins ambulatoires des cinq autres centres régionaux. Nous préconisons également le recours à l'approche employée dans le présent projet pour optimiser les rôles infirmiers dans d'autres cliniques. Tant à l'échelon régional que provincial, les intervenants devraient mettre en pratique les résultats du projet dans leurs discussions de planification stratégique et opérationnelle qui visent à améliorer les services de santé prodigués aux patients et à offrir du perfectionnement professionnel au personnel de première ligne. 
Tableau 3 : Extrait de la grille de référence des rôles et compétences

\begin{tabular}{|c|c|c|c|c|}
\hline \multicolumn{2}{|c|}{ Éléments de référence du rôle fonctionnel } & \multicolumn{3}{|l|}{ Normes } \\
\hline Tâches & Description & $\begin{array}{l}\text { BCCNP - infirmière } \\
\text { autorisée }\end{array}$ & $\begin{array}{l}\text { BCCNP - infirmière } \\
\text { auxiliaire }\end{array}$ & $\begin{array}{l}\text { C Norme de l'ACIO/ } \\
\text { CANO }\end{array}$ \\
\hline $\begin{array}{l}\text { Évaluation de } \\
\text { l'immunothérapie } \\
\text { (traitement } \\
\text { systémique) }\end{array}$ & $\begin{array}{l}\text { - Évaluation complète de } \\
\text { l'immunothérapie en } \\
\text { fonction du protocole de } \\
\text { traitement du patient et } \\
\text { des algorithmes de soutien } \\
\text { correspondants }\end{array}$ & $\begin{array}{l}\text { - Norme } 1: \text { indicateurs de } \\
\text { pratique clinique } 2,4 \\
\text { - Norme } 2: \text { indicateurs de } \\
\text { pratique clinique - tous } \\
\text { - Norme } 3: \text { indicateurs de } \\
\text { pratique clinique } 1,3,4 \\
\text { - Norme } 4: \text { indicateurs } \\
\text { de pratique clinique } 1,7 \\
10,13\end{array}$ & $\begin{array}{l}\text { - Norme } 1 \text { : indicateurs } 2, \\
\text { 4, 5, 6, 7, } 8 \\
\text { - Norme } 2 \text { : indicateurs } \\
\text { 1, 2, 3, 4, 5, 6, 7, 8, 9, } \\
\text { 10,11 } \\
\text { - Norme } 3 \text { : indicateurs } 1, \\
2,5,6,7,8 \\
\text { - Norme } 4: \text { indicateurs } 5 \text {, } \\
7,10,11\end{array}$ & $\begin{array}{l}\text { - Domaine 1: } \\
\text { compétences 1b), 1c), } \\
\text { 1d), 1e) } \\
\text { - Domaine 3: } \\
\text { compétences 3a), 3b) } \\
\text { - Domaine } 4 \text { : } \\
\text { compétences 4a), 4b) } \\
\text { - Domaine 5: } \\
\text { compétences 5e) } \\
\text { - Domaine 7: } \\
\text { compétences 7a) }\end{array}$ \\
\hline $\begin{array}{l}\text { Aiguillage vers les } \\
\text { soins palliatifs } \\
\text { (radiothérapie } \\
\text { et traitement } \\
\text { systémique) }\end{array}$ & $\begin{array}{l}\text { - Faciliter la conversation sur } \\
\text { les soins palliatifs avec le } \\
\text { patient et la famille } \\
\text { - Remplir et envoyer les } \\
\text { documents du programme } \\
\text { de soins palliatifs } \\
\text { - Formulaire de demande } \\
\text { - Avantages de la médication } \\
\text { - Volonté de décès à domicile } \\
\text { - Aiguillage vers les soins à } \\
\text { domicile } \\
\text { - Formulaires de planification } \\
\text { préalable des soins }\end{array}$ & $\begin{array}{l}\text { - Norme } 1: \text { indicateur de } \\
\text { pratique clinique } 4 \\
\text { - Norme } 2: \text { indicateurs de } \\
\text { pratique clinique } 2,3,6, \\
7,8,10,11,13 \\
\text { - Norme } 3: \text { indicateurs de } \\
\text { pratique clinique } 1,2,3, \\
4,6,9,10 \\
\text { - Norme } 4: \text { indicateurs } \\
\text { de pratique clinique } 1,2 \\
5,6,7,8,9,10,11,12,13\end{array}$ & $\begin{array}{l}\text { - Norme } 1: \text { indicateur } 7 \\
\text { - Norme } 2 \text { : indicateurs } 2 \text {, } \\
3,6,7,11 \\
\text { - Norme } 3 \text { : indicateurs } 1 \text {, } \\
\text { 2, 3, 5, 6, } 8 \\
\text { - Norme } 4: \text { indicateurs } \\
\\
5,6\end{array}$ & $\begin{array}{l}\text { - Domaine 2: } \\
\text { compétences 2i), 2j), } \\
\text { 2k), 2l; 7) } \\
\text { - Domaine } 3 \text { : } \\
\text { compétences 3a), 3b), } 3 \\
\text { (traitements palliatifs) } \\
\text { - Domaine } 4 \text { : } \\
\text { compétences - toutes } \\
\text { - Domaine 5: } \\
\text { compétences 5a), 5b), } \\
\text { 5c), 5e), 5f), 5g), 5i), } \\
\text { 5k) } \\
\text { - Domaine 6: } \\
\text { compétences - toutes } \\
\text { - Domaine 7: } \\
\text { compétence 7a) }\end{array}$ \\
\hline
\end{tabular}




\begin{tabular}{|c|c|c|c|c|}
\hline $\begin{array}{l}\text { Protocoles } \\
\text { d'enseignement aux } \\
\text { patients } \\
\text { (traitement } \\
\text { systémique) }\end{array}$ & $\begin{array}{l}\text { - Imprimer les documents à } \\
\text { remettre au patient sur les } \\
\text { médicaments inclus dans le } \\
\text { protocole } \\
\text { - Passer en revue ces } \\
\text { documents avec le patient }\end{array}$ & $\begin{array}{l}\text { - Norme } 1: \text { indicateur de } \\
\text { pratique clinique } 4 \\
\text { - Norme } 2: \text { indicateurs de } \\
\text { pratique clinique - tous } \\
\text { - Norme } 3: \text { indicateurs } \\
\text { de pratique clinique } 1,3 \text {, } \\
4,6,10 \\
\text { - Norme } 4: \text { indicateurs } \\
\text { de pratique clinique } 1,2 \text {, } \\
6,7,10,11,12,13\end{array}$ & $\begin{array}{l}\text { - Norme } 1: \text { indicateur } 7 \\
\text { - Norme } 2: \text { indicateurs } \\
\text { - tous } \\
\text { - Norme } 3: \text { indicateurs } 1 \text {, } \\
\text { 2, 3, 5, 6, } 8 \\
\text { - Norme } 4: \text { indicateur } 6\end{array}$ & $\begin{array}{l}\text { - Domaine 2: } \\
\text { compétence 2a) } \\
\text { - Domaine } 3 \text { : } \\
\text { compétences 3a), 3b), } 3 \\
\text { (traitement systémique } \\
\text { - a), 3b), } 3 \text { (traitement } \\
\text { systémique - c), 3d), } \\
3 \text { (autres traitements } \\
\text { - b), } 3 \text { (modalités } \\
\text { combinées), } 3 \\
\text { (traitements palliatifs), } 3 \\
\text { (gestion des symptômes } \\
\text { et des effets secondaires } \\
\text { - d), } 3 \text { (questions } \\
\text { de santé sexuelle - } \\
\text { a), } 3 \text { (questions de } \\
\text { santé sexuelle - b), } 3 \\
\text { (questions de santé } \\
\text { sexuelle - c) } \\
\text { - Domaine } 4 \text { : } \\
\text { compétences - toutes } \\
\text { - Domaine } 5: \\
\text { compétences } 5 c \text { c), 5e), } \\
5 \mathrm{~g} \text { ), } 5 \text { h), } 5 \mathrm{i} \text { ) } \\
\text { - Domaine } 6: \\
\text { compétences } 6 a \text { ), 6i) } \\
\text { - Domaine } 7: \\
\text { compétence } 7 a \text { ) }\end{array}$ \\
\hline $\begin{array}{l}\text { Documentation } \\
\text { (radiothérapie } \\
\text { et traitement } \\
\text { systémique) }\end{array}$ & $\begin{array}{l}\text { - Remplir la documentation } \\
\text { infirmière sur les } \\
\text { formulaires approuvés le } \\
\text { cas échéant pour toutes les } \\
\text { évaluations, les soins et les } \\
\text { interventions de prise en } \\
\text { charge des symptômes et } \\
\text { d'enseignement }\end{array}$ & $\begin{array}{l}\text { - Norme } 1: \text { indicateur de } \\
\text { pratique clinique } 4 \\
\text { - Norme } 2: \text { indicateurs de } \\
\text { pratique clinique } 7,11 \\
\text { - Norme } 3: \text { indicateurs de } \\
\text { pratique clinique } 1,2,3 \text {, } \\
4,6,9,10 \\
\text { - Norme } 4: \text { indicateurs } \\
\text { de pratique clinique } 1,2 \text {, } \\
3,4,5,6,7,8,9,10,13\end{array}$ & $\begin{array}{l}\text { - Norme } 1: \text { indicateur } 7 \\
\text { - Norme } 2: \text { indicateur } 6 \\
\text { - Norme } 3: \text { indicateurs } 1 \text {, } \\
\text { 2, 3, 5, 6, } 8 \\
\text { - Norme } 4: \text { indicateurs } \\
\text { 5, } 6\end{array}$ & $\begin{array}{l}\text { - Domaine de pratique } 2 \text { : } \\
\text { compétence } 2 \mathrm{e} \text { ) } \\
\text { - Domaine de pratique } 6 \text { : } \\
\text { compétence } 6 \mathrm{k} \text { ) }\end{array}$ \\
\hline \multicolumn{5}{|c|}{$\begin{array}{l}\text { Tableau 3. Extrait de la grille des rôles et compétences pour les cliniques de radiothérapie et de traitement systémique des unités de soins } \\
\text { ambulatoires. À des fins de référence, le codage alphanumérique des indicateurs et compétences correspond aux éléments listés dans les normes } \\
\text { du BCCNP et de l'ACIO/CANO. }\end{array}$} \\
\hline
\end{tabular}




\section{CONCLUSION}

Afin de clarifier le travail des infirmières et d'expliciter la portée de leur pratique et les compétences spécialisées requises pour répondre le mieux possible aux besoins des patients et de leur famille, il est essentiel de définir et d'optimiser le rôle de l'infirmière en oncologie dans l'unité de soins ambulatoires. Toutefois, très peu de recherches formelles se sont penchées sur l'optimisation du rôle de l'infirmière en oncologie dans ce milieu particulier. Dans la littérature

\section{RÉFÉRENCES}

Azar, J. M., Johnson, C. S., Frame, A. M., Perkins, S. M., Cottingham, A. H., \& Litzelman, D. K. (2017). Evaluation of interprofessional relational coordination and patients' perception of care in outpatient oncology teams. Journal of Interprofessional Care, 31(2), 273-276. https://doi.org/10.1080/13561820.2016.1248815

Berglund, C. B., \& Gustafsson, E., Johansson, H., \& Bergenmar, M. (2015). Nurse-led outpatient clinics in oncology care - Patient satisfaction, information and continuity of care. European Journal of Oncology Nursing : The Official Journal of European Oncology Nursing Society, 19(6), 724-730.

BC Cancer (2018). BC Cancer Nursing Chemotherapy and Biotherapy Certification Program. http://www.bccancer.bc.ca/ health-professionals/education-development/nursing/ chemotherapy-certification-program

BC Children's \& Women's Hospital, BC Cancer, UBC Nurse Researchers (2018). Care Team Design Initiative. BC Cancer Internal Document Version 2, April 2018.

BC Government (2019). Information Privacy \& Security (FOIPPA). https://www2.gov.bc.ca/gov/content/governments/policiesfor-government/bcea-policy-and-procedure-manual/foippa/ information-privacy-and-security-foippa

BCCNP (2018a). Professional Standards for Licensed Practical Nurses. https://www.bccnp.ca/Standards/LPN/StandardResources/LPN_ ProfessionalStandards.pdf

BCCNP (2018b). Professional Standards for Registered Nurses and Nurse Practitioners. https://www.bccnp.ca/Standards/RN_NP/ StandardResources/RN_NP_ProfessionalStandards.pdf

BCCNP (2018c). Scope of Practice for Licensed Practical Nurses. https:// www.bccnp.ca/Standards/LPN/StandardResources/LPN_ ScopeOfPractice.pdf

BCCNP (2018d). Scope of Practice for Registered Nurses. https:// www.bccnp.ca/Standards/RN_NP/StandardResources/RN_ ScopeofPractice.pdf

Canadian Cancer Society (2018). Canadian Cancer Statistics: A 2018 Special Report on cancer incidence by stage. http://www.cancer.ca/ / media/cancer.ca/CW/cancer\%20information/cancer\%20101/ Canadian\%20cancer\%20statistics/Canadian-Cancer-Statistics2018-EN.pdf?la=en

Canadian Nurses Association (2012). Staff Mix: Decision-making Framework for Quality Care. https://cna-aiic.ca/ /media/cna/pagecontent/pdf-en/staff_mix_framework_2012_e.pdf

CANO-ACIO (2006). Practice Standards and Competencies for the Specialized Oncology Nurse. https://cdn.ymaws.com/ www.cano-acio.ca/resource/resmgr/standards/CONEP_ Standards2006September.pdf existante sur l'optimisation, différents thèmes ont été relevés, notamment le rôle de l'infirmière dans l'équipe interdisciplinaire, les dimensions des soins centrés sur le patient et la pratique professionnelle des soins infirmiers oncologiques. S'il serait bien sûr souhaitable d'approfondir la recherche dans ce domaine, le présent projet d'amélioration de la qualité, en explorant et en précisant le rôle infirmier dans l'équipe interdisciplinaire, fournit tout de même aux infirmières en oncologie un cadre d'application de leurs normes de pratique professionnelle adapté aux besoins des patients.

CANO-ACIO (2017). Standards and Competencies for Cancer Chemotherapy Nursing Practice. https://cdn.ymaws.com/www.canoacio.ca/resource/resmgr/Resources/EN_CANO_Chemotherapy_ Standar.pdf

CANO-ACIO (2018). Radiation Oncology Nursing Practice Standards and Competencies. https://cdn.ymaws.com/www.cano-acio.ca/ resource/resmgr/standards/ronp_s\&c_web(2).pdf

Haas, S., Swan, B. A., \& Haynes, T. (2013). Developing ambulatory care registered nurse competencies for care coordination and transition management. Nursing Economic\$, 31(1), 44-43. http:// search.ebscohost.com/login.aspx?direct=true $\& \mathrm{db}=\mathrm{ccm} \& A N=1080$ $26607 \&$ site=ehost-live

Haas, S. A., Vlasses, F., \& Havey, J. (2016). Developing staffing models to support population health management and quality outcomes in ambulatory care settings. Nursing Economics, 34(3), 126-133. http://ezproxy.library.ubc.ca/login?url=https://search-proquestcom.ezproxy.library.ubc.ca/docview/1794904355?accountid=14656

Hagen B., O’Beorme M., Desai S., Stingl, M., Pachnowski C. A., \& Hayward S. (2007). Innovations in the ethical review of healthrelated quality improvement and research: The Alberta Research Ethics Community Consensus Initiative (ARECCI). Healthcare Policy, 2(4), 2-12. https://www.ncbi.nlm.nih.gov/pmc/articles/ PMC2585461/pdf/policy-02-e164.pdf

Health Professions Act (2018a). Nurses (registered) and nurse practitioners regulation. http://www.bclaws.ca/civix/document/id/ $\mathrm{crbc} / \mathrm{crbc} / 2842008$

Health Professions Act (2018b). Nurses (licensed practical) regulation. http://www.bclaws.ca/civix/document/id/crbc/ crbc/224_2015

Kaunonen, M., Salin, S., \& Aalto, P. (2015). Database nurse staffing indicators: Explaining risks of staff job dissatisfaction in outpatient care. Journal of Nursing Management, 23(5), 546-556. https://doi. org/10.1111/jonm.12169

Lee, C. T., Doran, D. M., Tourangeau, A. E., \& Fleshner, N. E. (2014). Perceived quality of interprofessional interactions between physicians and nurses in oncology outpatient clinics. European Journal of Oncology Nursing, 18, 619-625 https://doi-org.ezproxy. library.ubc.ca/10.1016/j.ejon.2014.06.004

More, L. (2017). Transition to practice - Part 2 implementing an ambulatory care registered nurse residency program: Competency - it's not just a task. Nursing Economic\$, 35(6), 317-326. http:// search.ebscohost.com/login.aspx?direct=true\&db=ccm\&AN=1268 $65182 \&$ site=ehost-live 